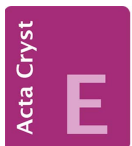
COMMUNICATIONS

ISSN 2056-9890

\section{Crystal structure of 3,4-dimethoxyphenol}

Heather A. Mills-Robles, ${ }^{\text {a }}$ Vasumathi Desikan, ${ }^{\text {a James A. }}$ Golen $^{b}$ and David R. Manke ${ }^{\text {b* }}$

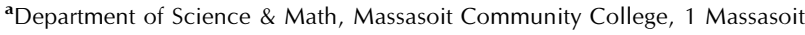
Boulevard, Brockton, MA 02302, USA, and ${ }^{\mathbf{b}}$ Department of Chemistry and Biochemistry, University of Massachusetts Dartmouth, 285 Old Westport Road, North Dartmouth, MA 02747, USA. *Correspondence e-mail: dmanke@umassd.edu

Received 20 November 2015; accepted 29 November 2015

Edited by K. Fejfarova, Institute of Macromolecular Chemistry, AS CR, v.v.i, Czech Republic

The title compound, $\mathrm{C}_{8} \mathrm{H}_{10} \mathrm{O}_{3}$, has two planar molecules in the asymmetric unit possessing mean deviations from planarity of 0.051 and $0.071 \AA$. In the crystal, there are two distinct infinite chains, both along [010]. The chains are formed by $\mathrm{O}-\mathrm{H} \cdots \mathrm{O}$ interactions between the phenol and both the 3-methoxy and the 4-methoxy groups.

Keywords: crystal structure; hydrogen bonding; phenols.

CCDC reference: 1439495

\section{Related literature}

For the crystal structure of the related 4-[(2,3-dimethylbut-3en-2-yl)oxy]-3-methoxyphenol, see: Yamamoto et al. (2014). For the crystal structure of 3,4,5-trimethoxyphenol, see: Jia et al. (2012). For background and crystal structures solved during the study, see: McDonald et al. (2015); Nguyen et al. (2015).

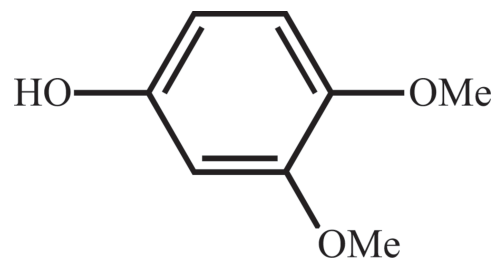

\section{Experimental}

\subsection{Crystal data}

$\mathrm{C}_{8} \mathrm{H}_{10} \mathrm{O}_{3}$

$M_{r}=154.16$

Orthorhombic, $\mathrm{Pbca}$

$a=8.7477$ (4) ^

$b=13.8218(7) \AA$

$c=26.6422(13) \AA$
$V=3221.3(3) \AA^{3}$

$Z=16$

Mo $K \alpha$ radiation

$\mu=0.10 \mathrm{~mm}^{-1}$

$T=120 \mathrm{~K}$

$0.5 \times 0.4 \times 0.4 \mathrm{~mm}$

\subsection{Data collection}

Bruker Venture D8 CMOS diffractometer

Absorption correction: multi-scan (SADABS; Bruker, 2014)

$T_{\min }=0.700, T_{\max }=0.746$

\subsection{Refinement}

$R\left[F^{2}>2 \sigma\left(F^{2}\right)\right]=0.042$

$w R\left(F^{2}\right)=0.116$

$S=1.04$

3996 reflections

205 parameters

2 restraints

Table 1

Hydrogen-bond geometry $\left(\AA{ }^{\circ}\right)$.

\begin{tabular}{lllll}
\hline$D-\mathrm{H} \cdots A$ & $D-\mathrm{H}$ & $\mathrm{H} \cdots A$ & $D \cdots A$ & $D-\mathrm{H} \cdots A$ \\
\hline $\mathrm{O} 1-\mathrm{H} 1 \cdots \mathrm{O}^{\mathrm{i}}$ & $0.86(1)$ & $2.25(1)$ & $2.9663(12)$ & $141(2)$ \\
$\mathrm{O}^{\mathrm{i}}-\mathrm{H} 1 \cdots \mathrm{O}^{\mathrm{i}}$ & $0.86(1)$ & $2.13(1)$ & $2.8834(13)$ & $145(2)$ \\
$\mathrm{O}^{2}-\mathrm{H} 4 \cdots \mathrm{O}^{\mathrm{i}}$ & $0.86(1)$ & $2.15(2)$ & $2.8384(13)$ & $137(2)$ \\
O4-H4 $^{\mathrm{O}} \mathrm{O}^{\mathrm{i}}$ & $0.86(1)$ & $2.37(1)$ & $3.1107(14)$ & $145(2)$ \\
\hline
\end{tabular}

Symmetry code: (i) $-x+\frac{1}{2}, y+\frac{1}{2}, z$.

Data collection: APEX2 (Bruker, 2014); cell refinement: SAINT (Bruker, 2014); data reduction: $S A I N T$; program(s) used to solve structure: SHELXS97 (Sheldrick, 2008); program(s) used to refine structure: SHELXL2014 (Sheldrick, 2015) and olex2.refine (Bourhis et al., 2015); molecular graphics: OLEX2 (Dolomanov et al., 2009); software used to prepare material for publication: OLEX2 and publCIF (Westrip, 2010).

\section{Acknowledgements}

We greatly acknowledge support from the National Science Foundation (CHE-1429086).

Supporting information for this paper is available from the IUCr electronic archives (Reference: FF2145).

\section{References}

Bourhis, L. J., Dolomanov, O. V., Gildea, R. J., Howard, J. A. K. \& Puschmann, H. (2015). Acta Cryst. A71, 59-75.

Bruker (2014). APEX2, SAINT, and SADABS. Bruker AXS Inc., Madison, Wisconsin, USA.

Dolomanov, O. V., Bourhis, L. J., Gildea, R. J., Howard, J. A. K. \& Puschmann, H. (2009). J. Appl. Cryst. 42, 339-341.

Jia, X.-C., Li, J., Yu, Z.-R., Zhang, H. \& Zhou, L. (2012). Acta Cryst. E68, o3160.

McDonald, K. J., Desikan, V., Golen, J. A. \& Manke, D. R. (2015). Acta Cryst. E71, 0406.

Nguyen, D. M., Desikan, V., Golen, J. A. \& Manke, D. R. (2015). Acta Cryst. E71, o533.

Sheldrick, G. M. (2008). Acta Cryst. A64, 112-122.

Sheldrick, G. M. (2015). Acta Cryst. C71, 3-8.

Westrip, S. P. (2010). J. Appl. Cryst. 43, 920-925.

Yamamoto, H., Ohkubo, K., Akimoto, S., Fukuzumi, S. \& Tsuda, A. (2014). Org. Biomol. Chem. 12, 7004-7017. 


\section{supporting information}

Acta Cryst. (2015). E71, o1019 [https://doi.org/10.1107/S2056989015022860]

\section{Crystal structure of 3,4-dimethoxyphenol}

\section{Heather A. Mills-Robles, Vasumathi Desikan, James A. Golen and David R. Manke}

\section{S1. Comment}

In a continuing collaborative study of the solid state structure of aromatic alcohols between UMass Dartmouth and Massasoit Community College (McDonald et al., 2015; Nguyen et al., 2015), we report herein the structure of 3,4-dimethoxyphenol. A similar 3,4-dialkoxyphenol complex has been structurally characterized (Yamamoto et al., 2014) and demonstrates tip-to-tail hydrogen bonding with the 4-alkoxy group. The structure of the trisubstituted 3,4,5-trimethoxyphenol demonstrates a similar interaction, with just the 4-methoxy group involved in hydrogen bonding (Jia et al., 2012). In contrast, the title compound exhibits hydrogen bonding chains with interactions involving the methoxy groups at both the 3 and 4 positions.

The molecular structure of the title compound is shown in Figure 1 .There are two molecules in the asymmetric unit, with non-hydrogen atoms possessing mean deviations from the plane of $0.051 \AA$ and $0.071 \AA$. There are two distinct hydrogen bonding chains which both propagate along [010]. One is formed by $\mathrm{O} 1-\mathrm{H} 1 \cdots \mathrm{O} 2$ and $\mathrm{O} 1-\mathrm{H} 1 \cdots \mathrm{O} 3$ interactions, and the other by $\mathrm{O} 4-\mathrm{H} 4 \cdots \mathrm{O} 5$ and $\mathrm{O} 4-\mathrm{H} 4 \cdots \mathrm{O} 6$ interactions. The packing of the title compound indicating hydrogen bonding is shown in Figure 2.

\section{S2. Experimental}

A commercial sample (Aldrich) was used for crystallization. Single crystals suitable for X-ray diffraction studies were grown by slow evaporation of a methylene chloride solution.

\section{S3. Refinement}

All non-hydrogen atoms were refined anisotropically (Olex2) by full matrix least squares on $\mathrm{F}^{2}$. Hydrogen atoms $\mathrm{H} 1$ and H4 were found from a Fourier difference map, and refined with a fixed distance of $0.86(0.005) \AA$ and isotropic displacement parameters of 1.50 times $U_{\mathrm{eq}}$ of the parent $\mathrm{O}$ atoms. The remaining hydrogen atoms were placed in calculated positions and then refined with a riding model with $\mathrm{C}-\mathrm{H}$ lengths of $0.95 \AA\left(\mathrm{sp}^{2}\right)$ and $0.98 \AA\left(\mathrm{sp}^{3}\right)$ with isotropic displacement parameters set to $1.20\left(\mathrm{sp}^{2}\right)$ and $1.50\left(\mathrm{sp}^{3}\right)$ times $U_{\text {eq }}$ of the parent $\mathrm{C}$ atom. 

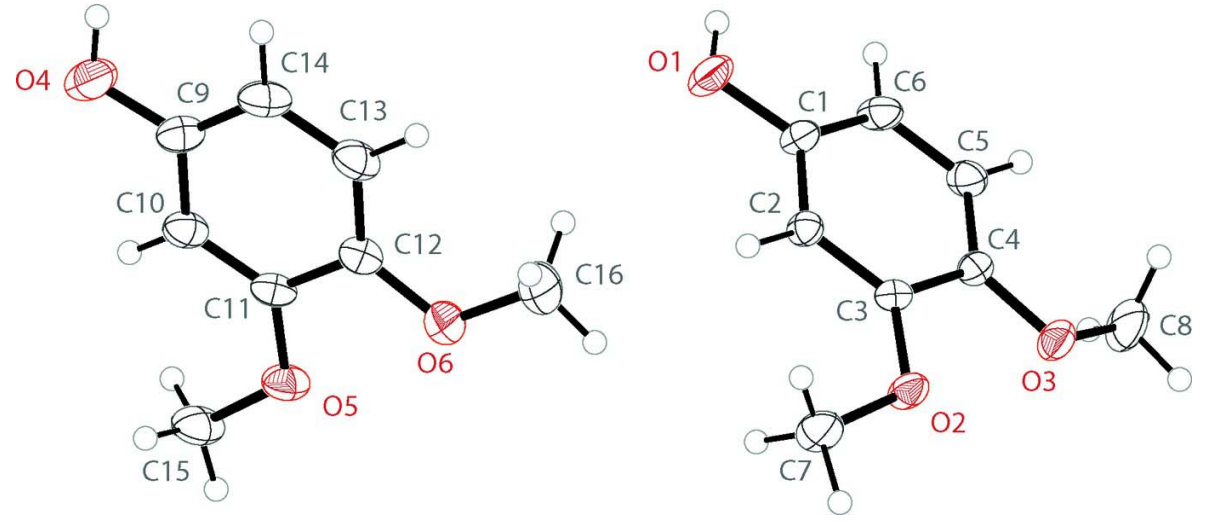

\section{Figure 1}

Molecular structure of the title compound, showing the atom-labelling scheme. Displacement ellipsoids are drawn at the $50 \%$ probability level. $\mathrm{H}$ atoms are drawn as spheres of arbitrary radius. 


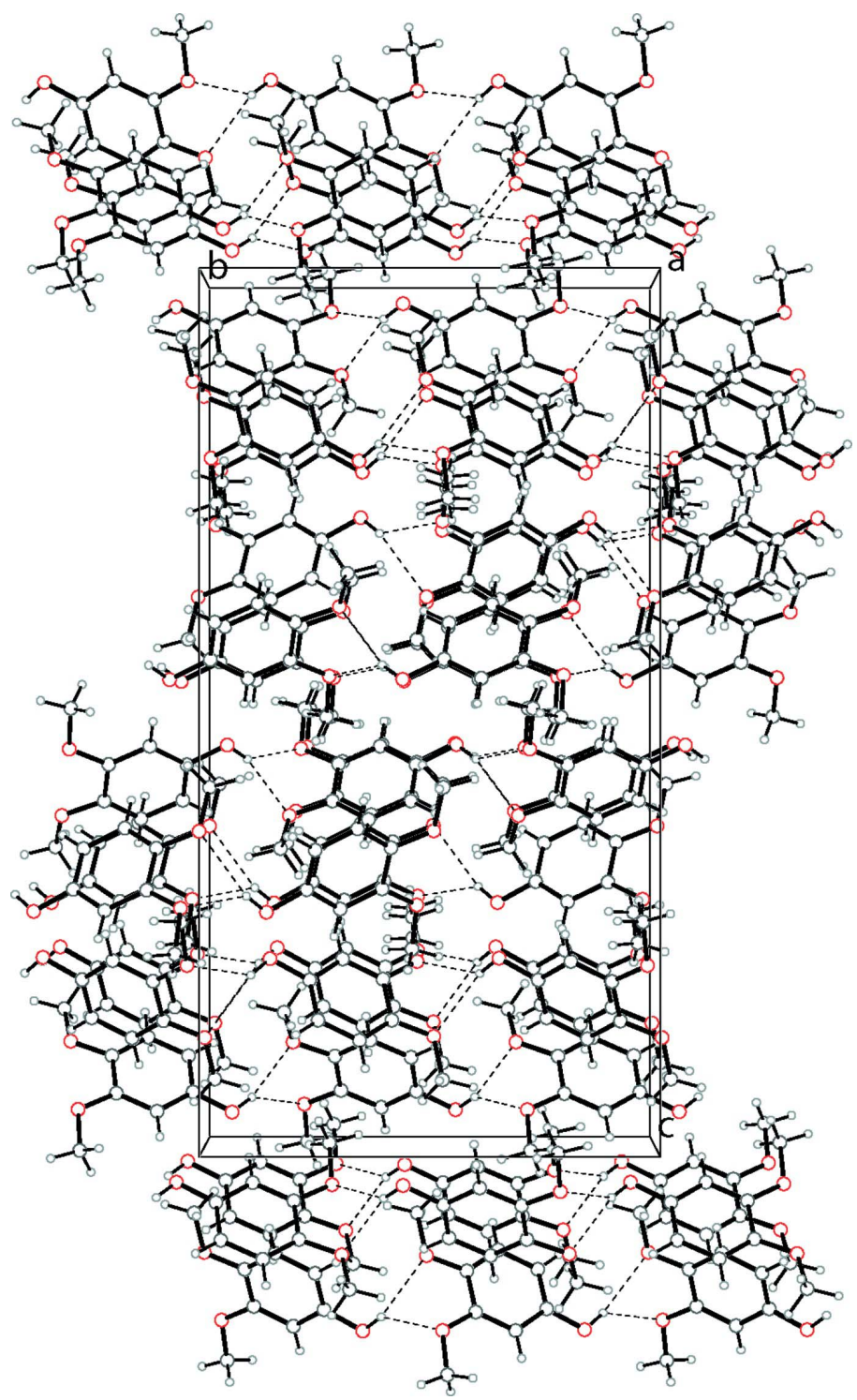

Figure 2

Molecular packing of the title compound with hydrogen bonding shown as dashed lines.

\section{3,4-Dimethoxyphenol}

\section{Crystal data}

$\mathrm{C}_{8} \mathrm{H}_{10} \mathrm{O}_{3}$

$M_{r}=154.16$

Orthorhombic, $\mathrm{Pbca}$

Hall symbol: -P 2ac $2 \mathrm{ab}$

$a=8.7477(4) \AA$

$b=13.8218(7) \AA$

$c=26.6422(13) \AA$

$V=3221.3(3) \AA^{3}$

$Z=16$
$F(000)=1312$

$D_{\mathrm{x}}=1.271 \mathrm{Mg} \mathrm{m}^{-3}$

Mo $K \alpha$ radiation, $\lambda=0.71073 \AA$

Cell parameters from 9890 reflections

$\theta=3.0-28.3^{\circ}$

$\mu=0.10 \mathrm{~mm}^{-1}$

$T=120 \mathrm{~K}$

Block, brown

$0.5 \times 0.4 \times 0.4 \mathrm{~mm}$ 


\begin{abstract}
Data collection
Bruker Venture D8 CMOS

diffractometer

Radiation source: Mo

TRIUMPH monochromator

$\varphi$ and $\omega$ scans

Absorption correction: multi-scan

(SADABS; Bruker, 2014)

$T_{\min }=0.700, T_{\max }=0.746$

29914 measured reflections

3996 independent reflections

3360 reflections with $I>2 \sigma(I)$

$R_{\text {int }}=0.032$

$\theta_{\text {max }}=28.4^{\circ}, \theta_{\min }=2.9^{\circ}$

$h=-11 \rightarrow 10$

$k=-18 \rightarrow 18$

$l=-35 \rightarrow 34$

\section{Refinement}

Refinement on $F^{2}$

Least-squares matrix: full

$R\left[F^{2}>2 \sigma\left(F^{2}\right)\right]=0.042$

$w R\left(F^{2}\right)=0.116$

$S=1.04$

3996 reflections

205 parameters

2 restraints

Hydrogen site location: mixed

$\mathrm{H}$ atoms treated by a mixture of independent

and constrained refinement

$w=1 /\left[\sigma^{2}\left(F_{\mathrm{o}}^{2}\right)+(0.0552 P)^{2}+1.1413 P\right]$

where $P=\left(F_{\mathrm{o}}^{2}+2 F_{\mathrm{c}}^{2}\right) / 3$

$(\Delta / \sigma)_{\max }=0.001$

$\Delta \rho_{\max }=0.25$ e $\AA^{-3}$

$\Delta \rho_{\min }=-0.25$ e $\AA^{-3}$

Special details

Experimental. Absorption correction: SADABS-2014/4 (Bruker,2014/4) was used for absorption correction. wR2(int) was 0.0791 before and 0.0531 after correction. The Ratio of minimum to maximum transmission is 0.9391 . The $\lambda / 2$ correction factor is 0.00150 .

Geometry. All esds (except the esd in the dihedral angle between two 1.s. planes) are estimated using the full covariance matrix. The cell esds are taken into account individually in the estimation of esds in distances, angles and torsion angles; correlations between esds in cell parameters are only used when they are defined by crystal symmetry. An approximate (isotropic) treatment of cell esds is used for estimating esds involving l.s. planes.
\end{abstract}

Fractional atomic coordinates and isotropic or equivalent isotropic displacement parameters $\left(\hat{A}^{2}\right)$

\begin{tabular}{lllll}
\hline & $x$ & $y$ & $z$ & $U_{\text {iso }} / U_{\text {eq }}$ \\
\hline O1 & $0.31642(12)$ & $0.55792(6)$ & $0.46341(4)$ & $0.0364(2)$ \\
H1 & $0.2656(18)$ & $0.6010(10)$ & $0.4472(6)$ & $0.055^{*}$ \\
O2 & $0.41171(10)$ & $0.21648(5)$ & $0.45901(3)$ & $0.0274(2)$ \\
O3 & $0.23732(11)$ & $0.19341(6)$ & $0.38304(3)$ & $0.0322(2)$ \\
C1 & $0.29128(13)$ & $0.46989(8)$ & $0.44100(4)$ & $0.0244(2)$ \\
C2 & $0.36772(12)$ & $0.39078(7)$ & $0.46188(4)$ & $0.0215(2)$ \\
H2 & 0.4337 & 0.3994 & 0.4899 & $0.026^{*}$ \\
C3 & $0.34642(12)$ & $0.29982(7)$ & $0.44142(4)$ & $0.0198(2)$ \\
C4 & $0.25063(13)$ & $0.28729(8)$ & $0.39961(4)$ & $0.0224(2)$ \\
C5 & $0.17669(14)$ & $0.36617(8)$ & $0.37932(4)$ & $0.0267(2)$ \\
H5 & 0.1123 & 0.3579 & 0.3509 & $0.032^{*}$ \\
C6 & $0.19567(14)$ & $0.45826(8)$ & $0.40017(5)$ & $0.0280(3)$ \\
H6 & 0.1433 & 0.5123 & 0.3864 & $0.034^{*}$ \\
C7 & $0.49570(17)$ & $0.22270(9)$ & $0.50473(5)$ & $0.0374(3)$ \\
H7A & 0.5365 & 0.1588 & 0.5133 & $0.056^{*}$ \\
H7B & 0.5803 & 0.2685 & 0.5006 & $0.056^{*}$ \\
H7C & 0.4280 & 0.2451 & 0.5317 & $0.056^{*}$ \\
C8 & $0.1208(2)$ & $0.17531(11)$ & $0.34658(6)$ & $0.0538(5)$ \\
H8A & 0.1214 & 0.1066 & 0.3375 & $0.081^{*}$
\end{tabular}




$\begin{array}{lllll}\text { H8B } & 0.0209 & 0.1924 & 0.3607 & 0.081^{*} \\ \text { H8C } & 0.1402 & 0.2145 & 0.3166 & 0.081^{*} \\ \text { O4 } & 0.27270(14) & 0.84893(7) & 0.72098(4) & 0.0463(3) \\ \text { H4 } & 0.319(2) & 0.8911(12) & 0.7029(7) & 0.069^{*} \\ \text { O5 } & 0.06921(10) & 0.52789(6) & 0.71498(3) & 0.0313(2) \\ \text { O6 } & 0.20065(12) & 0.49211(7) & 0.63132(3) & 0.0370(2) \\ \text { C9 } & 0.26184(15) & 0.76288(9) & 0.69618(5) & 0.0313(3) \\ \text { C10 } & 0.17021(14) & 0.69220(9) & 0.71881(5) & 0.0288(3) \\ \text { H10 } & 0.1198 & 0.7055 & 0.7496 & 0.035^{*} \\ \text { C11 } & 0.15354(13) & 0.60291(8) & 0.69608(4) & 0.0250(2) \\ \text { C12 } & 0.22572(14) & 0.58329(9) & 0.65010(4) & 0.0278(3) \\ \text { C13 } & 0.31638(15) & 0.65389(10) & 0.62848(5) & 0.0337(3) \\ \text { H13 } & 0.3660 & 0.6411 & 0.5975 & 0.040^{*} \\ \text { C14 } & 0.33589(15) & 0.74365(10) & 0.65161(5) & 0.0346(3) \\ \text { H14 } & 0.3998 & 0.7912 & 0.6367 & 0.042^{*} \\ \text { C15 } & 0.01260(18) & 0.53863(10) & 0.76497(5) & 0.0403(3) \\ \text { H15A } & -0.0456 & 0.4808 & 0.7744 & 0.060^{*} \\ \text { H15B } & 0.0986 & 0.5469 & 0.7881 & 0.060^{*} \\ \text { H15C } & -0.0540 & 0.5955 & 0.7667 & 0.060^{*} \\ \text { C16 } & 0.2624(2) & 0.47148(13) & 0.58306(6) & 0.0560(5) \\ \text { H16A } & 0.2374 & 0.4048 & 0.5737 & 0.084^{*} \\ \text { H16B } & 0.2187 & 0.5161 & 0.5583 & 0.084^{*} \\ \text { H16C } & 0.3737 & 0.4794 & 0.5839 & 0.084^{*} \\ \end{array}$

Atomic displacement parameters $\left(\AA^{2}\right)$

\begin{tabular}{lllllll}
\hline & $U^{11}$ & $U^{22}$ & $U^{33}$ & $U^{12}$ & $U^{13}$ & $U^{23}$ \\
\hline O1 & $0.0473(6)$ & $0.0169(4)$ & $0.0450(5)$ & $0.0030(4)$ & $-0.0158(4)$ & $-0.0031(4)$ \\
O2 & $0.0317(4)$ & $0.0177(4)$ & $0.0326(4)$ & $0.0028(3)$ & $-0.0104(3)$ & $0.0003(3)$ \\
O3 & $0.0436(5)$ & $0.0208(4)$ & $0.0322(4)$ & $-0.0027(4)$ & $-0.0134(4)$ & $-0.0036(3)$ \\
C1 & $0.0267(5)$ & $0.0170(5)$ & $0.0295(6)$ & $-0.0015(4)$ & $-0.0012(4)$ & $0.0002(4)$ \\
C2 & $0.0210(5)$ & $0.0201(5)$ & $0.0234(5)$ & $-0.0016(4)$ & $-0.0024(4)$ & $0.0003(4)$ \\
C3 & $0.0189(5)$ & $0.0179(5)$ & $0.0227(5)$ & $0.0003(4)$ & $0.0007(4)$ & $0.0030(4)$ \\
C4 & $0.0248(5)$ & $0.0195(5)$ & $0.0229(5)$ & $-0.0038(4)$ & $-0.0008(4)$ & $0.0002(4)$ \\
C5 & $0.0282(6)$ & $0.0260(5)$ & $0.0260(5)$ & $-0.0040(5)$ & $-0.0078(4)$ & $0.0042(4)$ \\
C6 & $0.0292(6)$ & $0.0212(5)$ & $0.0337(6)$ & $0.0008(4)$ & $-0.0067(5)$ & $0.0070(4)$ \\
C7 & $0.0450(7)$ & $0.0256(6)$ & $0.0416(7)$ & $0.0050(5)$ & $-0.0209(6)$ & $0.0027(5)$ \\
C8 & $0.0796(12)$ & $0.0334(7)$ & $0.0484(9)$ & $-0.0055(8)$ & $-0.0363(8)$ & $-0.0082(6)$ \\
O4 & $0.0618(7)$ & $0.0258(5)$ & $0.0513(6)$ & $-0.0033(5)$ & $0.0108(5)$ & $0.0018(4)$ \\
O5 & $0.0317(4)$ & $0.0287(4)$ & $0.0335(5)$ & $-0.0021(3)$ & $0.0081(4)$ & $0.0079(3)$ \\
O6 & $0.0491(6)$ & $0.0340(5)$ & $0.0279(4)$ & $-0.0052(4)$ & $0.0066(4)$ & $-0.0012(4)$ \\
C9 & $0.0318(6)$ & $0.0236(5)$ & $0.0385(6)$ & $0.0032(5)$ & $0.0006(5)$ & $0.0063(5)$ \\
C10 & $0.0289(6)$ & $0.0269(6)$ & $0.0306(6)$ & $0.0062(5)$ & $0.0052(5)$ & $0.0065(4)$ \\
C11 & $0.0207(5)$ & $0.0261(5)$ & $0.0283(6)$ & $0.0021(4)$ & $0.0008(4)$ & $0.0097(4)$ \\
C12 & $0.0277(6)$ & $0.0288(6)$ & $0.0269(6)$ & $0.0013(5)$ & $0.0000(4)$ & $0.0056(5)$ \\
C13 & $0.0346(7)$ & $0.0366(6)$ & $0.0300(6)$ & $0.0012(5)$ & $0.0094(5)$ & $0.0081(5)$ \\
C14 & $0.0313(6)$ & $0.0311(6)$ & $0.0413(7)$ & $-0.0012(5)$ & $0.0077(5)$ & $0.0124(5)$ \\
C15 & $0.0448(8)$ & $0.0339(6)$ & $0.0421(7)$ & $0.0050(6)$ & $0.0214(6)$ & $0.0106(6)$
\end{tabular}




$\begin{array}{lllllll}\mathrm{C} 16 & 0.0853(13) & 0.0480(9) & 0.0348(7) & -0.0094(9) & 0.0195(8) & -0.0073(7)\end{array}$

Geometric parameters $(\AA, \stackrel{o}{)}$

\begin{tabular}{|c|c|c|c|}
\hline $\mathrm{O} 1-\mathrm{H} 1$ & $0.859(5)$ & $\mathrm{O} 4-\mathrm{H} 4$ & $0.860(5)$ \\
\hline $\mathrm{O} 1-\mathrm{C} 1$ & $1.3731(13)$ & $\mathrm{O} 4-\mathrm{C} 9$ & $1.3638(16)$ \\
\hline $\mathrm{O} 2-\mathrm{C} 3$ & $1.3684(12)$ & $\mathrm{O} 5-\mathrm{C} 11$ & $1.3686(14)$ \\
\hline $\mathrm{O} 2-\mathrm{C} 7$ & $1.4251(14)$ & $\mathrm{O} 5-\mathrm{C} 15$ & $1.4285(15)$ \\
\hline $\mathrm{O} 3-\mathrm{C} 4$ & $1.3754(13)$ & $\mathrm{O} 6-\mathrm{C} 12$ & $1.3735(15)$ \\
\hline $\mathrm{O} 3-\mathrm{C} 8$ & $1.4302(16)$ & $\mathrm{O} 6-\mathrm{C} 16$ & $1.4236(17)$ \\
\hline $\mathrm{C} 1-\mathrm{C} 2$ & $1.3971(15)$ & $\mathrm{C} 9-\mathrm{C} 10$ & $1.4002(17)$ \\
\hline $\mathrm{C} 1-\mathrm{C} 6$ & $1.3816(16)$ & $\mathrm{C} 9-\mathrm{C} 14$ & $1.3786(18)$ \\
\hline $\mathrm{C} 2-\mathrm{H} 2$ & 0.9500 & $\mathrm{C} 10-\mathrm{H} 10$ & 0.9500 \\
\hline $\mathrm{C} 2-\mathrm{C} 3$ & $1.3829(14)$ & $\mathrm{C} 10-\mathrm{C} 11$ & $1.3824(17)$ \\
\hline $\mathrm{C} 3-\mathrm{C} 4$ & $1.4048(15)$ & $\mathrm{C} 11-\mathrm{C} 12$ & $1.4045(16)$ \\
\hline $\mathrm{C} 4-\mathrm{C} 5$ & $1.3781(16)$ & $\mathrm{C} 12-\mathrm{C} 13$ & $1.3831(17)$ \\
\hline $\mathrm{C} 5-\mathrm{H} 5$ & 0.9500 & $\mathrm{C} 13-\mathrm{H} 13$ & 0.9500 \\
\hline $\mathrm{C} 5-\mathrm{C} 6$ & $1.3986(16)$ & $\mathrm{C} 13-\mathrm{C} 14$ & 1.3957 (19) \\
\hline $\mathrm{C} 6-\mathrm{H} 6$ & 0.9500 & $\mathrm{C} 14-\mathrm{H} 14$ & 0.9500 \\
\hline C7-H7A & 0.9800 & C15-H15A & 0.9800 \\
\hline C7-H7B & 0.9800 & C15-H15B & 0.9800 \\
\hline $\mathrm{C} 7-\mathrm{H} 7 \mathrm{C}$ & 0.9800 & $\mathrm{C} 15-\mathrm{H} 15 \mathrm{C}$ & 0.9800 \\
\hline $\mathrm{C} 8-\mathrm{H} 8 \mathrm{~A}$ & 0.9800 & $\mathrm{C} 16-\mathrm{H} 16 \mathrm{~A}$ & 0.9800 \\
\hline $\mathrm{C} 8-\mathrm{H} 8 \mathrm{~B}$ & 0.9800 & C16-H16B & 0.9800 \\
\hline $\mathrm{C} 8-\mathrm{H} 8 \mathrm{C}$ & 0.9800 & $\mathrm{C} 16-\mathrm{H} 16 \mathrm{C}$ & 0.9800 \\
\hline $\mathrm{C} 1-\mathrm{O} 1-\mathrm{H} 1$ & $108.2(13)$ & $\mathrm{C} 9-\mathrm{O} 4-\mathrm{H} 4$ & $110.7(14)$ \\
\hline $\mathrm{C} 3-\mathrm{O} 2-\mathrm{C} 7$ & $117.19(9)$ & $\mathrm{C} 11-\mathrm{O} 5-\mathrm{C} 15$ & $116.82(10)$ \\
\hline $\mathrm{C} 4-\mathrm{O} 3-\mathrm{C} 8$ & $116.31(10)$ & $\mathrm{C} 12-\mathrm{O} 6-\mathrm{C} 16$ & $116.88(11)$ \\
\hline $\mathrm{O} 1-\mathrm{C} 1-\mathrm{C} 2$ & $116.34(10)$ & $\mathrm{O} 4-\mathrm{C} 9-\mathrm{C} 10$ & $116.09(11)$ \\
\hline $\mathrm{O} 1-\mathrm{C} 1-\mathrm{C} 6$ & $122.85(10)$ & $\mathrm{O} 4-\mathrm{C} 9-\mathrm{C} 14$ & $123.55(12)$ \\
\hline $\mathrm{C} 6-\mathrm{C} 1-\mathrm{C} 2$ & $120.81(10)$ & $\mathrm{C} 14-\mathrm{C} 9-\mathrm{C} 10$ & $120.35(12)$ \\
\hline $\mathrm{C} 1-\mathrm{C} 2-\mathrm{H} 2$ & 120.3 & $\mathrm{C} 9-\mathrm{C} 10-\mathrm{H} 10$ & 120.2 \\
\hline $\mathrm{C} 3-\mathrm{C} 2-\mathrm{C} 1$ & $119.35(10)$ & $\mathrm{C} 11-\mathrm{C} 10-\mathrm{C} 9$ & $119.65(11)$ \\
\hline $\mathrm{C} 3-\mathrm{C} 2-\mathrm{H} 2$ & 120.3 & $\mathrm{C} 11-\mathrm{C} 10-\mathrm{H} 10$ & 120.2 \\
\hline $\mathrm{O} 2-\mathrm{C} 3-\mathrm{C} 2$ & $125.04(9)$ & $\mathrm{O} 5-\mathrm{C} 11-\mathrm{C} 10$ & $124.89(11)$ \\
\hline $\mathrm{O} 2-\mathrm{C} 3-\mathrm{C} 4$ & $114.63(9)$ & $\mathrm{O} 5-\mathrm{C} 11-\mathrm{C} 12$ & $114.65(10)$ \\
\hline $\mathrm{C} 2-\mathrm{C} 3-\mathrm{C} 4$ & $120.33(9)$ & $\mathrm{C} 10-\mathrm{C} 11-\mathrm{C} 12$ & $120.47(11)$ \\
\hline $\mathrm{O} 3-\mathrm{C} 4-\mathrm{C} 3$ & $114.92(9)$ & $\mathrm{O} 6-\mathrm{C} 12-\mathrm{C} 11$ & $115.02(10)$ \\
\hline $\mathrm{O} 3-\mathrm{C} 4-\mathrm{C} 5$ & $125.51(10)$ & $\mathrm{O} 6-\mathrm{C} 12-\mathrm{C} 13$ & $125.96(11)$ \\
\hline $\mathrm{C} 5-\mathrm{C} 4-\mathrm{C} 3$ & $119.56(10)$ & $\mathrm{C} 13-\mathrm{C} 12-\mathrm{C} 11$ & $119.00(11)$ \\
\hline $\mathrm{C} 4-\mathrm{C} 5-\mathrm{H} 5$ & 119.7 & $\mathrm{C} 12-\mathrm{C} 13-\mathrm{H} 13$ & 119.6 \\
\hline $\mathrm{C} 4-\mathrm{C} 5-\mathrm{C} 6$ & $120.57(10)$ & $\mathrm{C} 12-\mathrm{C} 13-\mathrm{C} 14$ & $120.89(12)$ \\
\hline $\mathrm{C} 6-\mathrm{C} 5-\mathrm{H} 5$ & 119.7 & $\mathrm{C} 14-\mathrm{C} 13-\mathrm{H} 13$ & 119.6 \\
\hline $\mathrm{C} 1-\mathrm{C} 6-\mathrm{C} 5$ & $119.37(10)$ & $\mathrm{C} 9-\mathrm{C} 14-\mathrm{C} 13$ & $119.62(11)$ \\
\hline $\mathrm{C} 1-\mathrm{C} 6-\mathrm{H} 6$ & 120.3 & $\mathrm{C} 9-\mathrm{C} 14-\mathrm{H} 14$ & 120.2 \\
\hline $\mathrm{C} 5-\mathrm{C} 6-\mathrm{H} 6$ & 120.3 & C13-C14-H14 & 120.2 \\
\hline $\mathrm{O} 2-\mathrm{C} 7-\mathrm{H} 7 \mathrm{~A}$ & 109.5 & $\mathrm{O} 5-\mathrm{C} 15-\mathrm{H} 15 \mathrm{~A}$ & 109.5 \\
\hline
\end{tabular}




\begin{tabular}{|c|c|c|c|}
\hline $\mathrm{O} 2-\mathrm{C} 7-\mathrm{H} 7 \mathrm{~B}$ & 109.5 & $\mathrm{O} 5-\mathrm{C} 15-\mathrm{H} 15 \mathrm{~B}$ & 109.5 \\
\hline $\mathrm{O} 2-\mathrm{C} 7-\mathrm{H} 7 \mathrm{C}$ & 109.5 & $\mathrm{O} 5-\mathrm{C} 15-\mathrm{H} 15 \mathrm{C}$ & 109.5 \\
\hline $\mathrm{H} 7 \mathrm{~A}-\mathrm{C} 7-\mathrm{H} 7 \mathrm{~B}$ & 109.5 & $\mathrm{H} 15 \mathrm{~A}-\mathrm{C} 15-\mathrm{H} 15 \mathrm{~B}$ & 109.5 \\
\hline $\mathrm{H} 7 \mathrm{~A}-\mathrm{C} 7-\mathrm{H} 7 \mathrm{C}$ & 109.5 & $\mathrm{H} 15 \mathrm{~A}-\mathrm{C} 15-\mathrm{H} 15 \mathrm{C}$ & 109.5 \\
\hline $\mathrm{H} 7 \mathrm{~B}-\mathrm{C} 7-\mathrm{H} 7 \mathrm{C}$ & 109.5 & $\mathrm{H} 15 \mathrm{~B}-\mathrm{C} 15-\mathrm{H} 15 \mathrm{C}$ & 109.5 \\
\hline $\mathrm{O} 3-\mathrm{C} 8-\mathrm{H} 8 \mathrm{~A}$ & 109.5 & $\mathrm{O} 6-\mathrm{C} 16-\mathrm{H} 16 \mathrm{~A}$ & 109.5 \\
\hline $\mathrm{O} 3-\mathrm{C} 8-\mathrm{H} 8 \mathrm{~B}$ & 109.5 & $\mathrm{O} 6-\mathrm{C} 16-\mathrm{H} 16 \mathrm{~B}$ & 109.5 \\
\hline $\mathrm{O} 3-\mathrm{C} 8-\mathrm{H} 8 \mathrm{C}$ & 109.5 & $\mathrm{O} 6-\mathrm{C} 16-\mathrm{H} 16 \mathrm{C}$ & 109.5 \\
\hline $\mathrm{H} 8 \mathrm{~A}-\mathrm{C} 8-\mathrm{H} 8 \mathrm{~B}$ & 109.5 & $\mathrm{H} 16 \mathrm{~A}-\mathrm{C} 16-\mathrm{H} 16 \mathrm{~B}$ & 109.5 \\
\hline $\mathrm{H} 8 \mathrm{~A}-\mathrm{C} 8-\mathrm{H} 8 \mathrm{C}$ & 109.5 & $\mathrm{H} 16 \mathrm{~A}-\mathrm{C} 16-\mathrm{H} 16 \mathrm{C}$ & 109.5 \\
\hline $\mathrm{H} 8 \mathrm{~B}-\mathrm{C} 8-\mathrm{H} 8 \mathrm{C}$ & 109.5 & $\mathrm{H} 16 \mathrm{~B}-\mathrm{C} 16-\mathrm{H} 16 \mathrm{C}$ & 109.5 \\
\hline $\mathrm{O} 1-\mathrm{C} 1-\mathrm{C} 2-\mathrm{C} 3$ & $179.40(10)$ & $\mathrm{O} 4-\mathrm{C} 9-\mathrm{C} 10-\mathrm{C} 11$ & $-179.88(11)$ \\
\hline $\mathrm{O} 1-\mathrm{C} 1-\mathrm{C} 6-\mathrm{C} 5$ & $179.69(11)$ & $\mathrm{O} 4-\mathrm{C} 9-\mathrm{C} 14-\mathrm{C} 13$ & $-179.08(12)$ \\
\hline $\mathrm{O} 2-\mathrm{C} 3-\mathrm{C} 4-\mathrm{O} 3$ & $0.15(14)$ & $\mathrm{O} 5-\mathrm{C} 11-\mathrm{C} 12-\mathrm{O} 6$ & $-0.06(15)$ \\
\hline $\mathrm{O} 2-\mathrm{C} 3-\mathrm{C} 4-\mathrm{C} 5$ & $178.86(10)$ & $\mathrm{O} 5-\mathrm{C} 11-\mathrm{C} 12-\mathrm{C} 13$ & $-178.59(11)$ \\
\hline $\mathrm{O} 3-\mathrm{C} 4-\mathrm{C} 5-\mathrm{C} 6$ & $178.24(11)$ & $\mathrm{O} 6-\mathrm{C} 12-\mathrm{C} 13-\mathrm{C} 14$ & $-178.68(12)$ \\
\hline $\mathrm{C} 1-\mathrm{C} 2-\mathrm{C} 3-\mathrm{O} 2$ & $-178.48(10)$ & $\mathrm{C} 9-\mathrm{C} 10-\mathrm{C} 11-\mathrm{O} 5$ & $178.92(11)$ \\
\hline $\mathrm{C} 1-\mathrm{C} 2-\mathrm{C} 3-\mathrm{C} 4$ & $0.94(16)$ & $\mathrm{C} 9-\mathrm{C} 10-\mathrm{C} 11-\mathrm{C} 12$ & $-1.08(17)$ \\
\hline $\mathrm{C} 2-\mathrm{C} 1-\mathrm{C} 6-\mathrm{C} 5$ & $-0.61(18)$ & $\mathrm{C} 10-\mathrm{C} 9-\mathrm{C} 14-\mathrm{C} 13$ & $1.42(19)$ \\
\hline $\mathrm{C} 2-\mathrm{C} 3-\mathrm{C} 4-\mathrm{O} 3$ & $-179.33(10)$ & $\mathrm{C} 10-\mathrm{C} 11-\mathrm{C} 12-\mathrm{O} 6$ & $179.94(11)$ \\
\hline $\mathrm{C} 2-\mathrm{C} 3-\mathrm{C} 4-\mathrm{C} 5$ & $-0.62(16)$ & $\mathrm{C} 10-\mathrm{C} 11-\mathrm{C} 12-\mathrm{C} 13$ & $1.41(17)$ \\
\hline $\mathrm{C} 3-\mathrm{C} 4-\mathrm{C} 5-\mathrm{C} 6$ & $-0.32(18)$ & $\mathrm{C} 11-\mathrm{C} 12-\mathrm{C} 13-\mathrm{C} 14$ & $-0.32(19)$ \\
\hline $\mathrm{C} 4-\mathrm{C} 5-\mathrm{C} 6-\mathrm{C} 1$ & $0.93(18)$ & $\mathrm{C} 12-\mathrm{C} 13-\mathrm{C} 14-\mathrm{C} 9$ & $-1.1(2)$ \\
\hline $\mathrm{C} 6-\mathrm{C} 1-\mathrm{C} 2-\mathrm{C} 3$ & $-0.32(17)$ & $\mathrm{C} 14-\mathrm{C} 9-\mathrm{C} 10-\mathrm{C} 11$ & $-0.34(19)$ \\
\hline $\mathrm{C} 7-\mathrm{O} 2-\mathrm{C} 3-\mathrm{C} 2$ & $6.52(16)$ & $\mathrm{C} 15-\mathrm{O} 5-\mathrm{C} 11-\mathrm{C} 10$ & $-9.16(17)$ \\
\hline $\mathrm{C} 7-\mathrm{O} 2-\mathrm{C} 3-\mathrm{C} 4$ & $-172.93(11)$ & $\mathrm{C} 15-\mathrm{O} 5-\mathrm{C} 11-\mathrm{C} 12$ & $170.84(11)$ \\
\hline $\mathrm{C} 8-\mathrm{O} 3-\mathrm{C} 4-\mathrm{C} 3$ & $169.24(12)$ & $\mathrm{C} 16-\mathrm{O} 6-\mathrm{C} 12-\mathrm{C} 11$ & $175.56(13)$ \\
\hline $\mathrm{C} 8-\mathrm{O} 3-\mathrm{C} 4-\mathrm{C} 5$ & $-9.38(18)$ & $\mathrm{C} 16-\mathrm{O} 6-\mathrm{C} 12-\mathrm{C} 13$ & $-6.0(2)$ \\
\hline
\end{tabular}

Hydrogen-bond geometry $\left(\AA,{ }^{\circ}\right)$

\begin{tabular}{lllll}
\hline$D-\mathrm{H} \cdots A$ & $D-\mathrm{H}$ & $\mathrm{H} \cdots A$ & $D \cdots A$ & $D-\mathrm{H} \cdots A$ \\
\hline $\mathrm{O} 1-\mathrm{H} 1 \cdots \mathrm{O} 2^{\mathrm{i}}$ & $0.86(1)$ & $2.25(1)$ & $2.9663(12)$ & $141(2)$ \\
$\mathrm{O} 1-\mathrm{H} 1 \cdots 3^{\mathrm{i}}$ & $0.86(1)$ & $2.13(1)$ & $2.8834(13)$ & $145(2)$ \\
$\mathrm{O} 4-\mathrm{H} 4 \cdots 5^{\mathrm{i}}$ & $0.86(1)$ & $2.15(2)$ & $2.8384(13)$ & $137(2)$ \\
$\mathrm{O} 4-\mathrm{H} 4 \cdots \mathrm{O}^{\mathrm{i}}$ & $0.86(1)$ & $2.37(1)$ & $3.1107(14)$ & $145(2)$ \\
\hline
\end{tabular}

Symmetry code: (i) $-x+1 / 2, y+1 / 2, z$. 